\title{
Intracellular Recording of Cardiomyocyte Action Potentials with Nanopatterned Volcano-Shaped Microelectrode Arrays
}

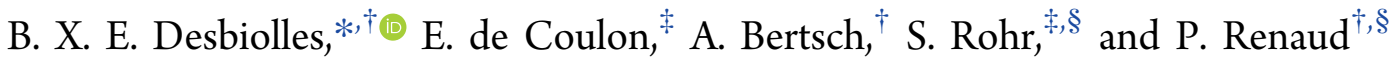 \\ ${ }^{\dagger}$ Laboratory of Microsystems LMIS4, Ecole Polytechnique Fédérale de Lausanne, 1015 Lausanne, Switzerland \\ ${ }^{\ddagger}$ Group Rohr, Department of Physiology, University of Bern, 3012 Bern, Switzerland
}

\section{Supporting Information}

\begin{abstract}
Micronanotechnology-based multielectrode arrays have led to remarkable progress in the field of transmembrane voltage recording of excitable cells. However, providing long-term optoporation- or electroporation-free intracellular access remains a considerable challenge. In this study, a novel type of nanopatterned volcano-shaped microelectrode (nanovolcano) is described that spontaneously fuses with the cell membrane and permits stable intracellular access. The complex nanostructure was manufactured following a simple and scalable fabrication process based on ion beam etching redeposition. The resulting ring-shaped structure provided passive intracellular access to neonatal rat cardiomyocytes. Intracellular action potentials were successfully recorded in vitro from different devices, and continuous recording for more than $1 \mathrm{~h}$ was achieved. By reporting transmembrane action potentials at potentially high spatial resolution without the need to apply physical triggers, the nanovolcanoes show distinct advantages over multielectrode arrays for the assessment of electrophysiological characteristics of cardiomyocyte networks at the transmembrane voltage level over time.
\end{abstract}

KEYWORDS: Intracellular electrophysiology, transmembrane access, microelectrode arrays, continuous recording, cell-electrode interface, nanoscale patterning, neonatal rat cardiomyocytes, nanovolcano

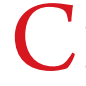
ardiovascular disease is the primary cause of morbidity and mortality worldwide and is responsible for more than $40 \%$ of the deaths in Europe alone. ${ }^{1}$ Among both disabling and fatal events, cardiac arrhythmias play a central role, and research efforts aimed at elucidating underlying mechanisms are extensive. They range from experiments at the subcellular level to experiments with large animals. Within this spectrum, electrophysiological studies performed at the single-cell and cell network levels form an important intermediate platform. ${ }^{2}$ Experimental approaches used for studies at the cellular and cell network levels include patch clamp recordings of single cells $^{3}$ and optical- ${ }^{4}$ or electrogram ${ }^{5,6}$ recordings for assessing cell network behavior in terms of action potential generation, propagation, and the establishment of reentrant activity. Electrogram recordings with extracellular multielectrode arrays (MEAs) are noninvasive and permit long-term measurements. They are primarily used for assessing network activity in cultured cell monolayers or slice preparations. Their disadvantage consists of the lack of reporting transmembrane voltage changes which are of interest when trying to relate specific activity patterns to changes in the action potential configuration and the occurrence of early or delayed postdepolarizations. This disadvantage is overcome by multisite optical recordings of transmembrane voltage with voltagesensitive dyes (VSDs) or voltage-sensitive fluorescent proteins (VSFPs; optogenetic reporters). ${ }^{7}$ Disadvantages of optical techniques include VSD phototoxicity that limits the duration of experiments to a few minutes ${ }^{8}$ and, for VSFPs, the circumstance that experiments are limited to tissues that have been genetically modified to express suitable optogenetic reporters of transmembrane voltage. Also, neither of these methods reports absolute values of transmembrane voltage.

Over the past decade, new approaches based on micronanotechnologies were developed with the aim of providing long-term intracellular recordings from cell networks that overcome the shortcomings of standard optical and MEA recording techniques. ${ }^{9-11}$ The proposed approaches consisted of a sharp nanopillar, ${ }^{12-16}$ a gold micromushroom, ${ }^{17,18}$ or plasmonic $^{19}$ electrode arrays, where the penetration of the sensing electrode into the cell is triggered by electro- or optoporation. With these techniques, the simultaneous intracellular recording of action potentials from cardiomyocyte networks could be obtained after the penetration-triggering event. Field-effect transistors mounted on nanowires and mechanically inserted inside electrogenic cells were shown to produce similar results. ${ }^{20-22}$

Until now, electrode geometries designed with the aim of permitting passive insertion into cell membranes have failed to

Received: May 30, 2019

Revised: $\quad$ August 9, 2019

Published: August 19, 2019 

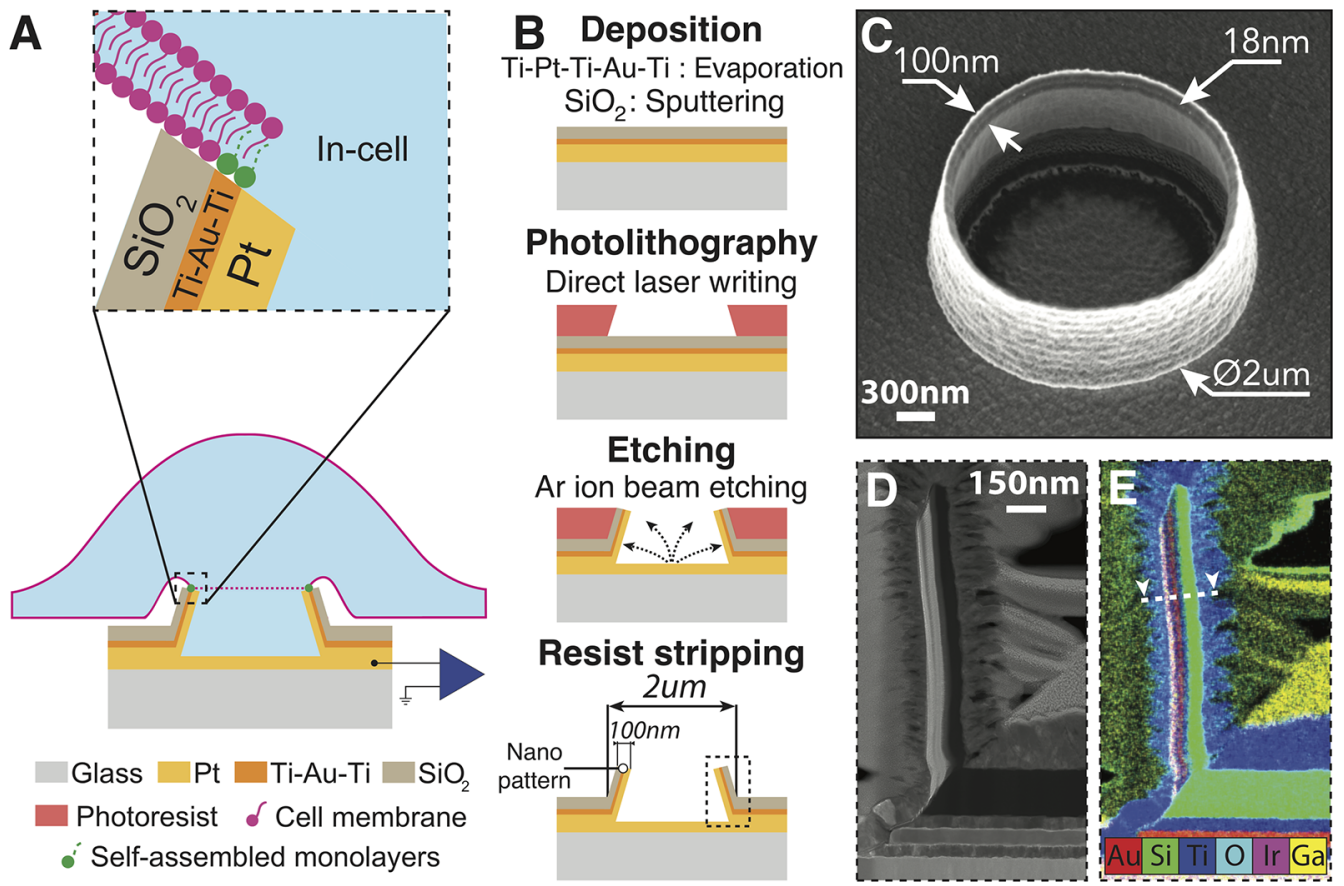

Figure 1. Concept and microfabrication of nanovolcanoes. (A) Schematic drawing showing the overall structure of the nanovolcano and the interface to a cell. The dashed lines represent the junctional cell membrane at the cell-electrode interface. (B) Microfabrication process flow used to manufacture the nanopatterned microelectrode. During ion beam etching, the material etched from the substrate is redeposited onto the photoresist side walls and the nanovolcano emerges after photoresist stripping. (C) SEM image of the microelectrode. (D and E) TEM image combined with EDX spectroscopy map of the redeposited multilayered wall. The thickness of each redeposited layer was measured along the dashed line.

produce a cell-electrode interface that is stable over extended periods of time. ${ }^{23}$ A promising approach that overcomes this problem consists of patterning nanoscale hydrophobic bands on otherwise hydrophilic substrates that allow the insertion of manufactured structures into cells. ${ }^{24-27}$ As demonstrated by VanderSarl et al., a planar electrode surrounded by an $\mathrm{Au}$ nanoring stacked in between two $\mathrm{Ti}$ layers which is functionalized with hydrophobic self-assembled monolayers produces gigaohm seals at the cell-electrode interface for several days. ${ }^{28}$

In the present study, a novel type of microelectrode that integrates the recent technological advances developed by Almquist et al. ${ }^{24}$ into an original structure is proposed to perform opto-/electroporation-free intracellular recording from cell networks. Because of its resemblance to a volcano, the novel nanopatterned microelectrode was named "nanovolcano", with "nano" standing for "nanopatterned". As illustrated in Figure 1A, the inner surface of the $2-\mu \mathrm{m}$-wide nanovolcano consists of a large conductive three-dimensional area made of $\mathrm{Pt}$ that ensures low access impedance of the intracellular space. The outside of the structure is covered with a 50-nm-thick insulating $\mathrm{SiO}_{2}$ layer that diminishes the current leak from the electrode. Furthermore, a 10- to 20-nm-thick $\mathrm{Au}$ nanoring, functionalized with self-assembled monolayers of alkanethiols and stacked in between two Ti layers, protrudes from the structure to fuse with the cell membrane, thereby optimizing the cell-electrode interface (Supporting Information, section 1). Finally, the 100-nm-thick multilayered nanovolcano wall has a shape similar to that of nanopillars that induce high-curvature regions in the cell membrane which maximize the chance of intracellular access. ${ }^{23,24,29,30}$

Neonatal rat cardiomyocytes were cultured for several days on the nanovolcano arrays, and electrophysiological recordings were used to investigate whether nanovolcanoes provide intracellular access to the cells. Signals recorded from up to $30 \%$ of the nanovolcanoes displayed the typical shape of rat cardiomyocyte action potentials, thereby demonstrating that the nanopatterned microstructures provided successful intracellular access to the cells.

\section{RESULTS}

1.1. Microfabrication. The nanovolcano was manufactured by exploiting the local redeposition on the photoresist side walls occurring during argon ion beam etching. ${ }^{31}$ As illustrated in Figure 1B, openings with a diameter of $2 \mu \mathrm{m}$ were patterned into a negative photoresist layer by standard photolithography, thereby exposing the multilayered $\mathrm{Ti}-\mathrm{Pt}-$ $\mathrm{Ti}-\mathrm{Au}-\mathrm{Ti}-\mathrm{SiO}_{2}$-coated glass substrate. During argon ion beam etching, part of the etched material from the substrate was redeposited on the side walls of the photoresist opening, which resulted in the buildup of the multilayered wall of the nanovolcano. The redeposition of the stacked layers of materials occurred in reverse order, starting from the topmost layer $\left(\mathrm{SiO}_{2}\right)$ and being stopped when the Pt layer was partially etched. A final resist stripping step completed the nanovolcano manufacturing process and resulted in the structure shown by the scanning electron microscope (SEM) image in Figure 1C. Following the formation of nanovolcanoes, 3- $\mu \mathrm{m}$-wide conductive tracks were patterned by conventional ion beam etching to independently interface each microelectrode to the peripheral connecting pads. To reduce capacitive current leaks from these tracks, a $4.5-\mu \mathrm{m}$-thick SU8 insulating layer was structured so as to result in $20-\mu \mathrm{m}$-wide openings around each nanovolcano. 


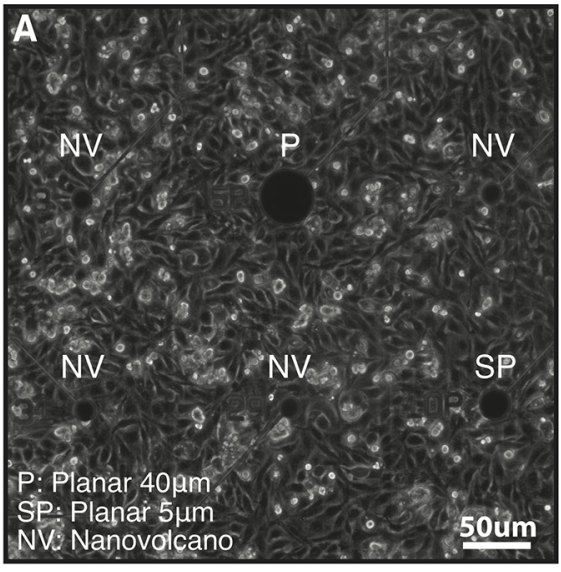

\section{B \\ Nanovolcano}
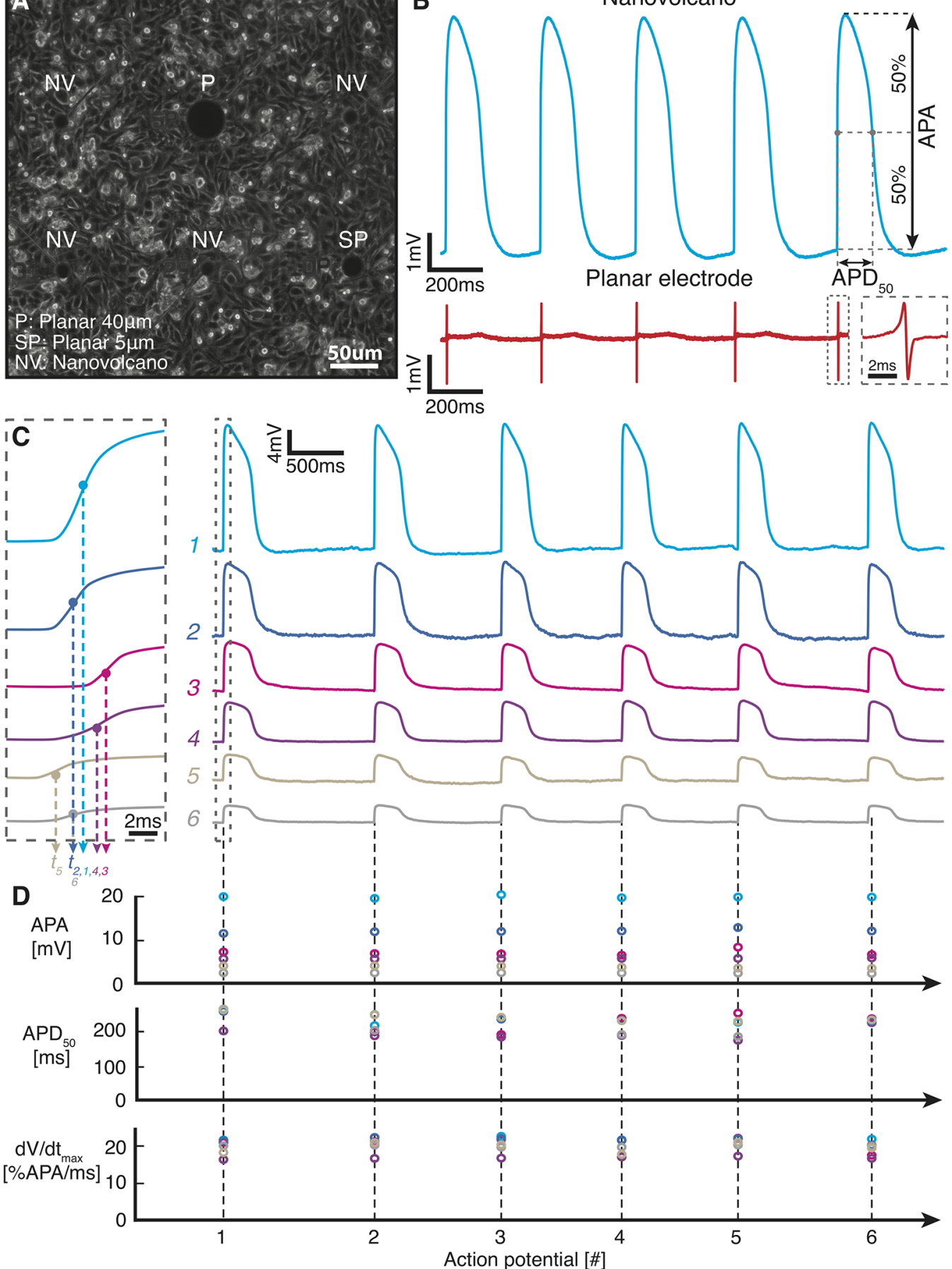

Figure 2. Intracellular recording of action potentials from primary rat cardiomyocyte monolayer. (A) Phase contrast image of a representative 3day-old primary rat cardiomyocyte monolayer culture grown on the nanovolcano array. (B) Intracellular recording of action potentials with a nanovolcano (upper trace) and simultaneously measured electrograms from a nearby planar electrode (lower trace) during spontaneous electrical activity (APA, action potential amplitude; $\mathrm{APD}_{50}$, action potential duration at $50 \%$ repolarization). (C) Simultaneous recording of spontaneous action potentials from six different channels of a nanovolcano array with action potential upstrokes shown at an expanded time scale on the left. Temporal shifts between activation midpoints indicate the presence of propagated electrical activity. (D) Temporal evolution of action potential parameters of the signals presented in $\mathrm{C}\left(\mathrm{d} V / \mathrm{d} t_{\max }\right.$ : maximal upstroke velocity).

The redeposited multilayered structure was characterized by transmission electron microscopy (TEM) combined with energy-dispersive X-ray spectroscopy (EDX). Figure 1D,E shows the redeposition of the etched substrate layers on the photoresist side walls during ion beam etching, with the topmost layer undergoing etching and redeposition first. The inner side of the wall is conductive and contiguous with the active electrode area, whereas the outer side $\left(\mathrm{SiO}_{2}\right)$ is insulating. (IrOx was used instead of Pt for TEM imaging in Figure 1E.) The two titanium layers on either side of the gold nanoring prevent its diffusion through the structure. The gold nanoring thickness was $18 \mathrm{~nm}$ as determined by EDX on a single line scan (cf. the dashed line in Figure 1E). The redeposition was uniform along the entire wall. 

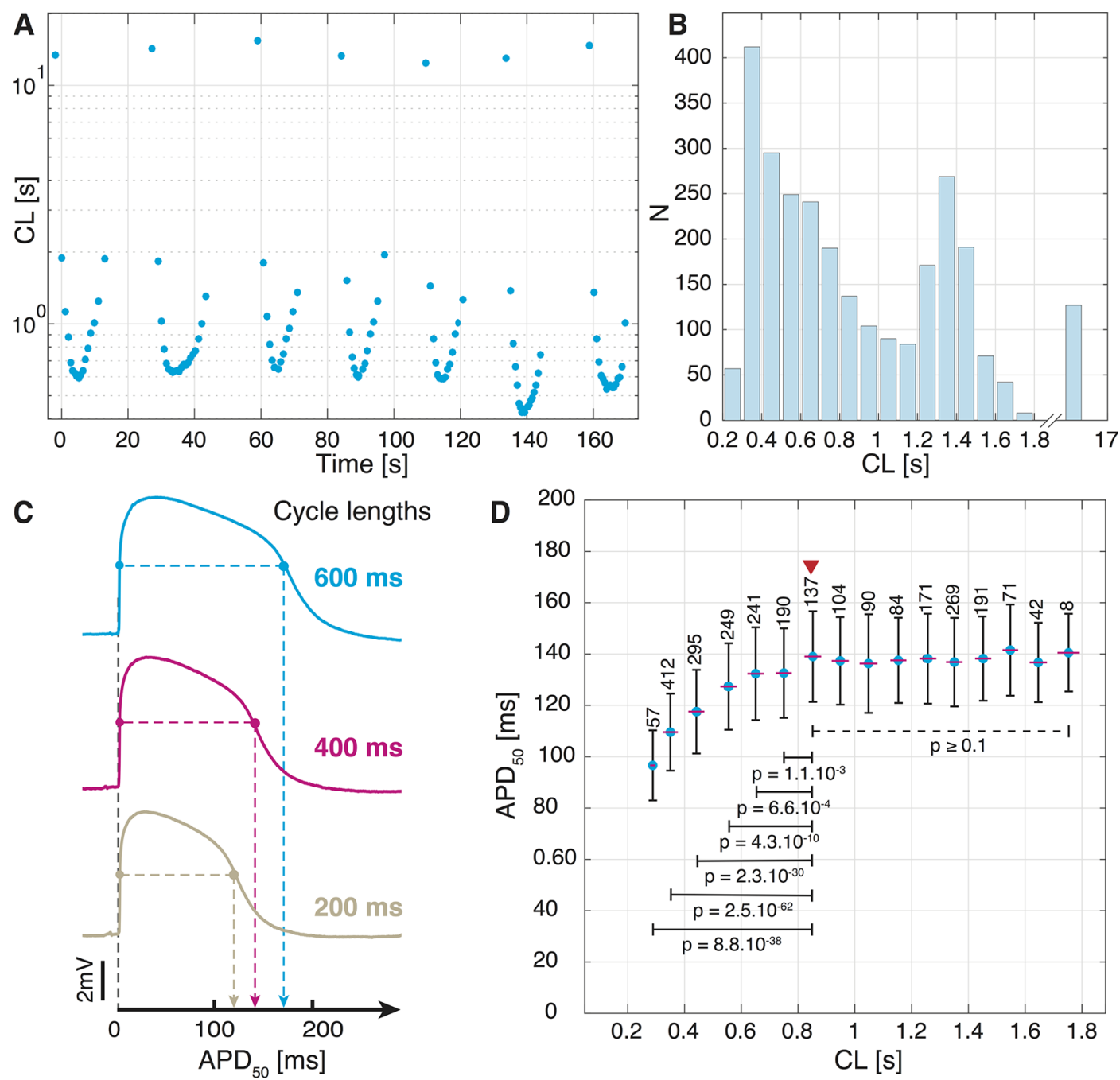

Figure 3. APD restitution. (A) Time course of cycle lengths (CL) of a spontaneously active preparation. (The 3 min fragment shown is typical for the entire recording.) (B) Distribution of CLs of the same preparation as determined during 60 min of continuous recording (bin width, $200 \mathrm{ms)}$. (C) Action potential shapes at different CLs. (D) Dependence of $\mathrm{APD}_{50}$ on CL (mean $\pm \mathrm{SD}$; is indicated above values; $p$ values are given with respect to the data point marked with a red triangle).

The nanovolcano arrays were composed of 32 electrodes: 1 Pt reference electrode, a stimulation dipole, six $40-\mu \mathrm{m}$-wide and two $5-\mu \mathrm{m}$-wide standard planar Pt electrodes, and $21 \mathrm{Pt}$ nanovolcanoes that were separated by a pitch of $300 \mu \mathrm{m}$. The overall layout of the array is depicted in the Supporting Information in section 2.

1.2. Intracellular Action Potential Recording from Networks of Cardiomyocytes with Nanovolcano Arrays. To validate the device, monolayers of neonatal rat ventricular cardiomyocytes were cultured on the nanopatterned array for 48 to $72 \mathrm{~h}$ prior to the electrophysiological recordings. As shown in Figure 2A, the cell monolayers displayed uniform adherence to the entire substrate including the region of the two standard planar electrodes and the four nanovolcanoes.

Figure $2 \mathrm{~B}$ shows a series of sequential intracellular action potentials (APs) recorded by a nanovolcano from a spontaneously active cardiomyocyte monolayer. The shape of the signal is typical of APs of cultured rat cardiomyocytes. ${ }^{32}$ Action potential upstrokes coincided, as expected, with extracellular field potentials (electrograms) recorded simultaneously by nearby planar extracellular electrodes of the array. Recordings of a series of intracellular APs obtained simultaneously from several nanovolcanoes of the same device are shown in Figure 2C with the corresponding AP parameters (amplitude, APA; duration at $50 \%$ repolarization, $\mathrm{APD}_{50}$, and maximal action potential upstroke velocity, $\left.\mathrm{d} V / \mathrm{d} t_{\max }\right)$ shown in Figure 2D. To ease comparison with conventionally measured values of $\mathrm{d} V / \mathrm{d} t_{\max }$, maximal upstroke velocities were calculated after normalization of the signal amplitudes (\% action potential amplitude, \%APA $/ \mathrm{ms}$ ). Under the assumption of a typical nonattenuated action potential amplitude of $100 \mathrm{mV}, 33 \%$ $\mathrm{APA} / \mathrm{ms}$ corresponds to the commonly used unit $\mathrm{V} / \mathrm{s}$. While APs recorded by different nanovolcanoes were similar with respect to $\mathrm{APD}_{50}(244.9 \pm 50 \mathrm{~ms}, N=147)$ and $\mathrm{d} V / \mathrm{d} t_{\max }$ $(21.7 \pm 2.7 \% \mathrm{APA} / \mathrm{ms}, N=147)$, they differed with respect to the amplitude which ranged from 1.5 to $20.3 \mathrm{mV}$. This variability is likely explained by differences in the coupling parameters of the nanovolcano to the cells. The differences in the midpoints of action potential upstrokes depicted in the expanded inset of Figure $2 \mathrm{C}$ demonstrate the presence of propagated electrical activity which is typical for cardiomyocyte monolayers.

1.3. Dependence of $A P D_{50}$ on the Frequency of Spontaneous Activity. The instantaneous frequency of 

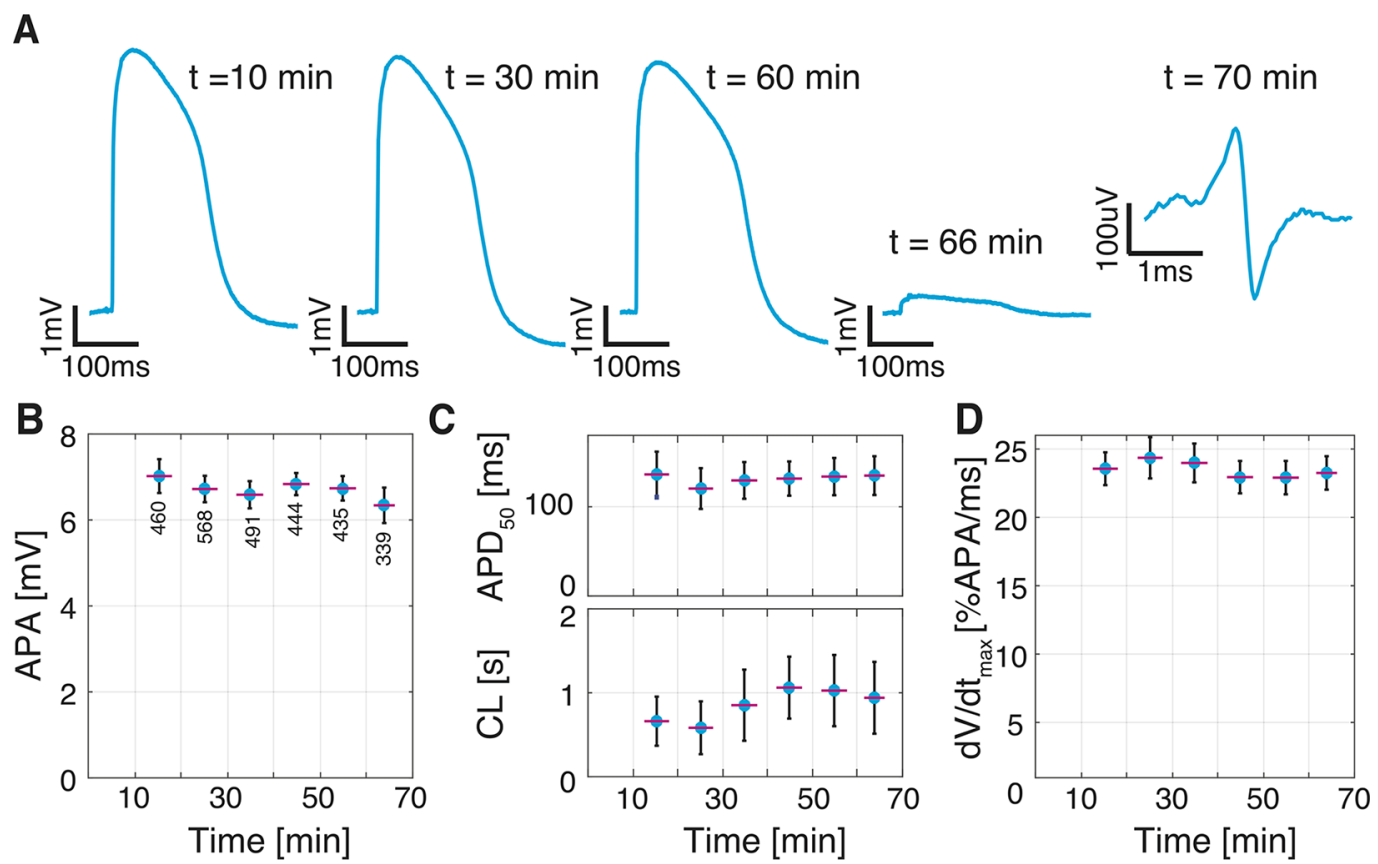

Figure 4. Change in action potential shape during continuous recordings by a nanovolcano. (A) Action potentials recorded at the times indicated. (B) Evolution of APA, (C) $\mathrm{APD}_{50}$ and CLs, and (D) $\mathrm{d} V / \mathrm{d} t_{\max }$ (mean $\pm \mathrm{SD}, N$ given below values in panel B).

spontaneous action potentials was assessed by measuring the period between successive action potentials (= cycle length, CL). CLs varied over time as shown in Figure $3 \mathrm{~A}$ for a $3 \mathrm{~min}$ fragment of a $60 \mathrm{~min}$ lasting recording. The distribution of CLs (range, $0.2-17 \mathrm{~s}$; bin width, $200 \mathrm{~ms}$ ) determined for the entire recording of $60 \mathrm{~min}$ is shown in Figure 3B. Differences in CLs permitted us to investigate whether nanovolcanoes are suited to detect frequency-dependent changes in $\mathrm{APD}_{50}$ (APD restitution) in order to further validate the physiological relevance of the recorded data. For this purpose, $\mathrm{APD}_{50}$ values of each AP were correlated to the preceding CL. As shown in Figure $3 \mathrm{C}$ for individual APs recorded by a single nanovolcano, $A P D_{50}$ values decreased with decreasing CL, thereby exhibiting the salient feature of $\mathrm{APD}$ restitution. When binning $\mathrm{APD}_{50}$ values of all signals are recorded from the same nanovolcano over the entire recording period, an increase in $\mathrm{APD}_{50}$ with increasing CL was observed that leveled off at CLs $>800 \mathrm{~ms}$ (Figure 3D). For the preparation shown, the change in $\mathrm{APD}_{50}$ ranged from $96.6 \pm 14 \mathrm{~ms}(\mathrm{CL}=287.7 \pm 9.3 \mathrm{~ms} ; N=57)$ to $139 \pm 18 \mathrm{~ms}(\mathrm{CL}=850.3 \mathrm{~ms} \pm 29.6 \mathrm{~ms} ; N=137 ; p=$ $\left.8.8 .10^{-38}\right)$, which reproduces findings reported before for APD restitution of neonatal rat cardiomyocyte monolayers using conventional measurement techniques. ${ }^{6}$

1.4. Stability of Nanovolcano Recordings. Nanovolcanoes permitted continuous long-term recordings for up to $1 \mathrm{~h}$ without appreciable changes in the general AP shape (Figure 4A; left three signals). The stability of the recordings is reflected by the analysis of $\mathrm{AP}$ parameters presented in Figure 4B,C,D. Although the APAs decreased by $9.7 \%$ from $7.02 \pm$ $0.39 \mathrm{mV}$ (interval $=10-20 \mathrm{~min} ; N=460$ ) to $6.34 \pm 0.41 \mathrm{mV}$ (interval $\left.=60-70 \mathrm{~min} ; N=339 ; p=3.21 \times 10^{-94}\right), \mathrm{APD}_{50}$ remained unchanged $(136.2 \pm 25.5 \mathrm{~ms}$ vs $134.9 \pm 21.7 \mathrm{~ms} ; p=$ 0.44). The transient decrease in $\mathrm{APD}_{50}$ between 20 and $30 \mathrm{~min}$ is likely explained by the simultaneously occurring transient increase in the frequency of spontaneous activity and, hence, APD restitution. Maximal upstroke velocities decreased by $1.3 \%$ from $23.56 \pm 1.20 \% \mathrm{APA} / \mathrm{ms}$ (interval $=10-20 \mathrm{~min}$ ) to
$23.25 \pm 1.22 \% \mathrm{APA} / \mathrm{ms}$ (interval $=60-70 \mathrm{~min} ; p=$ $\left.3.56 .10^{-4}\right)$. The absence of a change in $\mathrm{APD}_{50}$ and the moderate decline of APA and $\mathrm{d} V / \mathrm{d} t_{\max }$ over time indicate that cell viability was little, if any, affected by the nanovolcano recording. The final phase of the successful nanovolcano recording is illustrated by the two signals shown on the right of Figure 4A. It was heralded by a sudden drop in the APA $(t=$ $66 \mathrm{~min}$ ) followed by the appearance of extracellular field potentials $(t=70 \mathrm{~min})$, which likely indicates spontaneous membrane resealing. No coupling-uncoupling cycles were observed when reassessing the signals of nanovolcanoes $24 \mathrm{~h}$ after initial successful intracellular access.

\section{DISCUSSION}

The novel type of microelectrode developed in this work combines numerous recent technological advances into a single structure. By contrast to previous micronanoelectrodes, the high aspect ratio of the sharp nanovolcano wall permits in-cell access without limiting the active area of the recording electrode. ${ }^{9}$ This results in a relatively low electrode impedance (details in the Supporting Information, section 3) and, accordingly, high signal-to-noise ratios. The robustness of the cell-electrode interface is ensured by the electrically insulating walls preventing current leakage and by the functionalized nanoscale pattern that enables fusing of the nanovolcano to the cell membrane.

Manufacturing the multimaterial nanopatterned microstructure would be a major challenge when using conventional processes only. However, by exploiting ion beam etching redeposition, manufacturing can be simplified to a reliable and scalable four-step method that is compatible with complementary metal oxide semiconductor (CMOS) technologies. Accordingly, the nanovolcanoes can be manufactured directly on CMOS chips, thereby opening the perspective to be further developed into a self-standing intracellular electrophysiology platform. ${ }^{34-36}$ The fabrication process is simple and scalable and leads to homogeneous structures on the wafer scale. 
By varying the geometry of the photoresist openings, planar control electrodes with different dimensions were integrated into the device following the same fabrication process. Electrograms recorded by these electrodes were used as a baseline indicator of the presence of electrical activity and confirmed that planar electrodes having active areas similar to the nanovolcanoes permit artifact-free extracellular field potential (electrogram) recordings.

After seeding, primary neonatal rat ventricular cardiomyocytes showed a uniform attachment to the entire surface of the device and formed a well-coupled cell monolayer. The morphology and electrical activity of the cell monolayers cultured on the nanostructured substrate were not different from those of control monolayers grown on standard glass coverslips, thereby demonstrating the biocompatibility of the microstructure. ${ }^{37}$ Depending on the degree of contamination with cardiac myofibroblasts, cardiomyocyte monolayers were either quiescent or showed synchronized spontaneous activity. $^{38}$

The device presented demonstrates for the first time passive trans-membrane accesses to primary rat cardiomyocytes and therefore significantly improves upon previous micronanotechnology-based multielectrode arrays where electroporation, ${ }^{12-18}$ optoporation, ${ }^{19}$ or mechanical pressure ${ }^{20,21}$ needs to be applied to provide intracellular access. Moreover, by providing direct and stable recordings for more than $1 \mathrm{~h}$, nanovolcanoes achieved results comparable to those from state-of-the-art technologies in terms of the recording duration. $^{12,14,16}$

Overall, more than 3000 intracellular action potentials were successfully recorded from cardiomyocyte monolayer cultures grown on four different devices. The distributions of action potential amplitudes, recording durations, and yields of nanovolcanoes with successful in-cell access per device are summarized in the Supporting Information, section 5. Action potentials could be recorded simultaneously from up to $6 / 21$ nanovolcanoes per device. This maximal yield of nearly $30 \%$ suggests that arrays of nanovolcanoes are potentially suited for the spatially resolved assessment of transmembrane signals in networks of cardiomyocytes. Prospectively, this yield could be increased by optimizing the nanovolcano geometry (diameter, height, wall thickness, and inclination) as well as the nanopatterned self-assembled monolayers in order to maximize the penetration probability of the structure. Additionally, the spatial resolution of the device is limited only by the overall size of the microelectrode pad ( $30 \mu \mathrm{m}$ wide) and the electrical tracks ( $3 \mu \mathrm{m}$ wide). The microelectrode pad size could be decreased to the diameter of a single nanovolcano, thereby permitting significant increases in the microelectrode density and spatial resolution of the device.

Nanovolcano recordings of intracellular APs showed, with the exception of attenuated APAs, a typical AP shape as well as $\mathrm{APD}_{50}$ and $\mathrm{d} V / \mathrm{d} t_{\max }$ characteristics that match APs recorded using conventional sharp electrode or patch clamp techniques. ${ }^{33}$ Also, nanovolcanoes permitted the characterization of APD restitution, which illustrates the physiological relevance of this measurement approach. ${ }^{6}$

Even though attenuated, APAs reached amplitudes of as high as $20 \mathrm{mV}$ (20\% APA of an actual AP), which is significantly higher than amplitudes reported for state-of-theart micronanotechnology-based multielectrode arrays such as gold micromushrooms $(\max \mathrm{APA}=5 \mathrm{mV}),{ }^{17}$ plasmonic nanoelectrodes $(\max . \mathrm{APA}=1.8 \mathrm{mV}),{ }^{16}$ or metaelectrodes $(\max$ APA $=5 \mathrm{mV}) .{ }^{19}$ The superior performance of nanovolcanoes may be explained by the fact that they have access resistances of as low as $1.3 \mathrm{G} \Omega$ (Supporting Information, section 3 ), which is $>100$ times smaller than those of gold micromushrooms ${ }^{9}$ and is negligible compared to the input impedance of the amplifier used $(\sim 1 \mathrm{~T} \Omega)$. Accordingly, nanovolcanoes have the potential to record nonattenuated intracellular action potentials or even absolute resting membrane potentials if the ratio of junctional-to-seal resistance is further minimized. Such optimization may be achieved by varying the nanovolcano geometry as well as the composition of the self-assembled monolayers. The potential impact of these measures on the AP shape and amplitude is described in the Supporting Information, section 4.

Ideally, intracellular electrodes would permit uninterrupted long-term recordings of the electrical activity of networks of excitable cells. With nanovolcanoes, maximal recording periods were limited to slightly more than an hour. During this time, APA, $\mathrm{d} V / \mathrm{d} t_{\text {max }}$ and $\mathrm{APD}_{50}$ showed little if any change. In the example shown in Figure 4, this stable period was followed by a sudden and drastic decrease in APAs and, ultimately, the replacement of APs by electrogram-like signals. Given that the peak-to-peak amplitudes of these electrograms $(262 \pm 62 \mu \mathrm{V}$, $N=23$ ) were similar to electrograms recorded simultaneously by neighboring extracellular nanovolcanoes of the same device $(253 \pm 118 \mu \mathrm{V} ; N=45)$, a spontaneous closing of the cell membrane that restores the situation of nonintracellular nanovolcanoes seems plausible. The alternative explanation, a loss of the seal, cannot be ruled out. The investigation of the latter possibility is hampered by the fact that the seal resistance $\left(R_{\text {seal }}\right)$ is in series with the high charge transfer resistance $\left(R_{\mathrm{ct}}\right)$ of the nanovolcanoes, which precludes an accurate determination of a change in $R_{\text {seal }}$ during the loss of signal. While we have no direct evidence favoring a specific mechanism giving rise to spontaneous in-cell access by the nanovolcanoes, the high stress imposed on the cell membrane by the nanowall combined with an abrupt release of static charges accumulating at the electrode may explain the spontaneous process. In this context, the sharp geometry of the nanovolcano in conjunction with the nanopatterned self-assembled monolayers may maximize the chances of static charge-induced electroporation. If we further assume that a static discharge was preferably induced by turning on the amplifier system, this may explain why we never observed transitions from extracellular to intracellular recordings during our experiments. Conversely, resealing of the static-charge-induced pores reported by others $^{13,14}$ may explain the loss of intracellular access over time.

Our work agrees with previous studies showing that hollow structures significantly improve the recording duration compared to other structures aimed at gaining intracellular access. For instance, 3D hollow plasmonic electrodes were shown to provide intracellular access for up to $80 \mathrm{~min}$ after optoporation, ${ }^{16}$ and iridium oxide nanotube electrodes retained intracellular access for an hour after electroporation. By contrast, gold nanopillars lost in-cell access after 1 min. ${ }^{14}$ As demonstrated by Lin et al., the cell membrane curvature induced by hollow geometries increases the pore resealing time and, hence, the intracellular recording. ${ }^{14}$ As reported in this study, the nanovolcanoes perform similarly to hollow electrodes in regard to the duration of successful in-cell access, which supports the idea that hollow geometries delay pore closure. 
Finally, the low electrode impedance at low frequency permits a substantial charge injection into the cells and potentially render the nanovolcano a tool for creating new horizons in the field of intracellular amperometric sensing. ${ }^{39-42}$

\section{CONCLUSIONS}

In this work, we present a new type of nanopatterned microelectrode demonstrating significant advances compared to state-of-the-art microelectrodes for multisite intracellular electrophysiology. Improvements include a microstructure that enables spontaneous in-cell access and a self-assembled monolayer supporting stabilization of the cell-electrode interface. Furthermore, the structure features a large electrode area and hence a low impedance. Despite the complexity of the nanopatterned structure, the manufacturing of nanovolcanoes at the wafer scale is straightforward because it exploits nonconventional redeposition processes occurring during standard ion beam etching.

Proof of principle experiments with primary rat cardiomyocyte cell cultures demonstrate that the nanovolcanoes provide spontaneous intracellular access without the application of penetrating triggers such as opto- or electroporation. Upon gaining intracellular access, the nanovolcanoes successfully reported transmembrane voltage changes over extended periods of time. Apart from permitting the assessment of changes in AP shape in general, the nanovolcano recordings have the distinct advantage over extracellular multielectrode array recordings in that they allow for the investigation of highly pathological events such as early and delayed postdepolarizations that are central to cardiac arrhythmogenesis. This feature may render nanovolcanoes especially suited for pharmacological screening purposes aimed at detecting adverse cardiac side effects of novel compounds.

\section{METHODS}

4.1. Microfabrication. A standard glass float wafer ( 100 $\mathrm{mm}$ in diameter, $550 \mu \mathrm{m}$ thick) was covered by successive evaporations of $\mathrm{Ti}-\mathrm{Pt}-\mathrm{Ti}-\mathrm{Au}-\mathrm{Ti}(10 / 250 / 50 / 50 / 50 \mathrm{~nm})$ using an EVA760 e-beam evaporator (Alliance Concept, France), followed by an additional deposition of a 150-nmthick $\mathrm{SiO}_{2}$ layer performed with a Spider 600 sputter-coater (Pfeiffer Vacuum, France). Subsequently, the substrate was spin-coated with a $1.6-\mu \mathrm{m}$-thick AZnLof2020 photoresist layer (MicroChemicals, Germany) exploiting an EVG150 automatic spin-coater/developer (EVG, Austria), into which $2-\mu \mathrm{m}$ diameter openings were patterned with an i-line VPG200 direct laser writer (Heidelberg, Germany) operating at a dose of $80 \mathrm{~mJ} / \mathrm{cm}^{2}$. After a postexposure bake at $110^{\circ} \mathrm{C}$ with a 50 $\mu \mathrm{m}$ proximity gap for $75 \mathrm{~s}$, the wafer was developed for $46 \mathrm{~s}$ using an AZ 726 MIF commercial developer (MicroChemicals, Germany) dispensed using an EVG150 coater/developer. The sample was then bombarded with $\mathrm{Ar}^{+}$ions at an angle of incidence of $0^{\circ}$ using an IBE350 ion beam etcher (Veeco, USA). During this step, materials from the substrate were etched and redeposited on the photoresist side walls. The photoresist was finally stripped using $500 \mathrm{~W} \mathrm{O}$ plasma $\left(\mathrm{O}_{2}\right.$ flow $400 \mathrm{~mL} / \mathrm{min}$ ) for $7 \mathrm{~min}$ with a TePla 300 microwave plasma system (PVA TePla, Germany).

Electrically conductive tracks were patterned in a 3- $\mu \mathrm{m}$-thick AZ9221 photoresist layer (MicroChemicals) spin-coated with a Rite Track 88 series automatic coater (Rite Track, USA), exposed with a VPG200 direct laser writer at a dose of $280 \mathrm{~mJ} /$ $\mathrm{cm}^{2}$ and developed for $102 \mathrm{~s}$ using a Rite Track 88 series automatic developer (Rite Track). Thereafter, a 2 min reflow at $120{ }^{\circ} \mathrm{C}$ was performed on a Sawatec HP200 hot plate (Sawatec, Switzerland) prior to etching the substrate positioned at an angle of incidence of $-35^{\circ}$ with respect to the ion beam generated in an IBE350 ion beam etcher. The photoresist was stripped in $500 \mathrm{~W} \mathrm{O}_{2}$ plasma $\left(\mathrm{O}_{2}\right.$ flow 400 $\mathrm{mL} / \mathrm{min}$ ) for $7 \mathrm{~min}$ created by a TePla 300 microwave plasma system.

Finally, a $4.5-\mu \mathrm{m}$-thick GM1050 SU8 insulating layer (Gersteltec, Switzerland) was spin-coated using a Sawatec LSM250 manual coater (Sawatec, Switzerland) and soft baked at $130{ }^{\circ} \mathrm{C}$ for $5 \mathrm{~min}$ on a Sawatec HP200 hot plate. After a 20 $\mathrm{mJ} / \mathrm{cm}^{2}$ exposure using a VPG200 direct laser writer, the SU8 layer was postexposure baked on a Sawatec HP200 hot plate for $20 \mathrm{~min}$ at $85{ }^{\circ} \mathrm{C}$ before being manually developed in propylene glycol methyl ether acetate (PGMEA) for $1.5 \mathrm{~min}$ and rinsed for an additional minute in isopropanol. A final hard bake for $2 \mathrm{~h}$ at $135{ }^{\circ} \mathrm{C}$ on a Sawatec HP200 hot plate completed the fabrication process.

SEM images were taken with a Merlin SEM (Zeiss, Germany) at $1.5 \mathrm{kV}$ and $50 \mathrm{pA}$. Both the backscattered and secondary electrons were detected to enhance the contrast difference between the different layers of the wall. Scanning TEM and the corresponding EDX analysis were acquired with a Tecnai Osiris TEM (FEI, USA).

4.2. Device Preparation. The device was completed by mounting a glass ring onto the chip that formed the cell culture well using epoxy resin (Epo-Tek 302-3M; Epoxy Technology, USA). The microelectrode arrays were sterilized for $30 \mathrm{~s}$ with $\mathrm{O}_{2}$ plasma (100 W, 650 mTorr; Diener Electronic, Germany) before they were submerged for $30 \mathrm{~min}$ in a $20 \mathrm{mmol} / \mathrm{L}$ hexanethiol solution in pure ethanol for self-assembled monolayer formation. After ethanol rinsing, the chips were thoroughly rinsed in sterile deionized water. Prior to cell seeding, the substrate was coated with collagen type IV (C5533, Sigma, Germany). All recordings presented in this study were acquired using new devices. As discussed in the Supporting Information, section 6, the reuse of nanovolcano arrays is not recommended.

4.3. Isolation and Culture of Primary Rat Cardiomyocytes. Primary neonatal rat (Wistar, 1 day old) ventricular cardiomyocytes were obtained using established procedures. ${ }^{43}$ Experiments were performed in accordance with federal guidelines for animal experimentation under license BE27/17 of the state veterinary department of the canton Bern. In short, hearts from 8 to 10 neonatal rats were excised, and ventricles were trimmed in ice-cold Hank's balanced salt solution (HBSS) without $\mathrm{Ca}^{2+}$ and $\mathrm{Mg}^{2+}$ (3-02F29-I, BioConcept, Switzerland) containing trypsin (0.1\%; Sigma, T4674) and pancreatin $(120 \mu \mathrm{g} / \mathrm{mL} ; \mathrm{P}$ 3292, Sigma). Ventricles were minced into small tissue pieces and subjected to four to five consecutive dissociation steps in a stirred vessel at $36{ }^{\circ} \mathrm{C}$. After each step, the supernatant was collected and stored on ice and new dissociation solution was added to the pieces of tissue. The dissociated cells were centrifuged and resuspended in medium M199 containing Hank's salts (M7653, Sigma), penicillin $\left(20 \mathrm{U} / \mathrm{ml}\right.$; P7794, Sigma), vitamin $\mathrm{B}_{12}(2 \mu \mathrm{g} / \mathrm{mL}$; Sigma, V2876), bromodeoxyuridin $(100 \mu \mathrm{mol} / \mathrm{L} ; \mathrm{B}-5002$, Sigma), vitamin C (18 $\mu \mathrm{mol} / \mathrm{L} ; \mathrm{A}-4544$, Sigma $)$, epinephrine (10 $\mu \mathrm{mol} / \mathrm{L} ; \mathrm{E} 4250$, Sigma), L-glutamin $(680 \mu \mathrm{M} / \mathrm{L} ; \mathrm{G} 7513$, Sigma), and $10 \%$ neonatal calf serum (S 0125, Biochrom, Bioswisstec, Switzerland). Cardiomyocytes were enriched by 
preplating for $120 \mathrm{~min}$ in cell culture flasks. Cell numbers in the supernatant were determined using a hemocytometer and adjusted by dilution to result in a density of $\sim 3500$ cardiomyocytes per $\mathrm{mm}^{2}$ After seeding, the cultures were incubated at $36{ }^{\circ} \mathrm{C}$ in an atmosphere containing $0.8 \% \mathrm{CO}_{2}$. After $24 \mathrm{~h}$, the cell culture medium was renewed and the serum level was reduced to $5 \%$. Experiments were conducted 48 to 72 $\mathrm{h}$ postseeding. At this time, the cellular electrophysiology of cardiomyocytes has recovered from the isolation process and overgrowth with noncardiomyocytes such as myofibroblasts is still moderate.

4.4. Electrophysiology. Electrophysiological recordings with 48 to $72 \mathrm{~h}$ old cultures were performed in an incubator $\left(37{ }^{\circ} \mathrm{C}\right.$; $\left.0.8 \% \mathrm{CO}_{2}\right)$. A dc-coupled recording system (Digital Lynx SX with a HS36 head stage; Neuralynx, USA) was used to record intracellular action potentials and electrograms. Apart from bandwidth limitations imposed by the array and the recording system $\left(R_{\mathrm{in}}=1 \mathrm{~T} \Omega ; C_{\mathrm{in}}=2 \mathrm{pF}\right)$, the signals that are shown correspond to actual nonfiltered data.

\section{ASSOCIATED CONTENT}

\section{S Supporting Information}

The Supporting Information is available free of charge on the ACS Publications website at DOI: 10.1021/acs.nanolett.9b02209.

Detailed information regarding the fusion of the nanopattern with the cell membrane, the layout of the nanovolcano array, the experimental and theoretical characterization of the electrode-electrolyte interface, the cell-electrode interface modeling, and the distribution of the recording duration, signal amplitude, and yield (PDF)

\section{AUTHOR INFORMATION}

\section{Corresponding Author}

*E-mail: benoit.desbiolles@epfl.ch.

\section{ORCID}

\section{B. X. E. Desbiolles: 0000-0001-6848-3289}

\section{Author Contributions}

P.R. and B.X.E.D. conceived the project, B.X.E.D. manufactured the novel devices in a clean room. E.d.C. and S.R. handled the cardiomyocyte isolation and culture, B.X.E.D. and E.d.C. conducted the electrophysiological experiments, E.d.C., B.X.E.D., and S.R. interpreted the results, P.R. and A.B. supervised the technological part of the project, and S.R. supervised the biological part of the project. All authors discussed the results and contributed to the final manuscript.

\section{Author Contributions}

${ }^{\S}$ Shared last authorship.

\section{Notes}

The authors declare no competing financial interest.

\section{ACKNOWLEDGMENTS}

This work was partially funded by the Swiss National Science Foundation (grant numbers 200021-175943 and 310030169234). The authors thank the CMi (Center of Micronanotechnology), CIME (Centre Interdisciplinaire de Microscopie Electronique), AFA (Atelier de Fabrication Additive), and ACI (Atelier pour le routage et la fabrication de circuit imprimés) staff for their outstanding support and availability. Special thanks go to Regula Flückiger Labrada for her cell culture work, Christian Dellenbach for his development of the electronic recording and stimulation system (both at the Department of Physiology, University of Bern), Marc Heuschkel for his advice concerning the interfacing, Walid Amanhoud for his support with the particle swarm optimization, Shahriar Shalileh for his contribution to the theoretical model, and Nicolas Maino for his assistance in the laboratory. The authors sincerely thank the anonymous reviewers whose comments helped us to greatly improve this manuscript.

\section{REFERENCES}

(1) Timmis, A.; et al. European Society of Cardiology: Cardiovascular disease statistics 2017. Eur. Heart J. 2018, 39, 508577.

(2) Stevenson, W. G. In Cardiac Electrophysiology: From Cell to Bedside; Zipes, D. P., Jalife, J., Eds.; Elsevier/Saunders: Philadelphia, PA, 2013.

(3) Sakmann, B. Patch Clamp Techniques for Studying Ionic Channels in Excitable Membranes. Annu. Rev. Physiol. 1984, 46, 455472.

(4) Rohr, S.; Kucera, J. P. Optical recording system based on a fiber optic image conduit: Assessment of microscopic activation patterns in cardiac tissue. Biophys. J. 1998, 75, 1062-1075.

(5) Kucera, J. P.; Heuschkel, M. O.; Renaud, P.; Rohr, S. Power-law behavior of beat-rate variability in monolayer cultures of neonatal rat ventricular myocytes. Circ. Res. 2000, 86, 1140-5.

(6) Imboden, M.; et al. High-speed Mechano-active MEA for Investigating Rapid Stretch Effects on Cardiac Tissue. Nat. Commun. 2019, 10, 834 .

(7) O’Shea, C.; et al. Cardiac optogenetics and optical mapping Overcoming spectral congestion in all-optical cardiac electrophysiology. Front. Physiol. 2019, 10, 182.

(8) Rohr, S.; Salzberg, B. M. Multiple site optical recording of transmembrane voltage (MSORTV) in patterned growth heart cell cultures: assessing electrical behavior, with microsecond resolution, on a cellular and subcellular scale. Biophys. J. 1994, 67, 1301-1315.

(9) Spira, M. E.; Hai, A. Multi-electrode array technologies for neuroscience and cardiology. Nat. Nanotechnol. 2013, 8, 83-94.

(10) Abbott, J.; Ye, T.; Ham, D.; Park, H. Optimizing Nanoelectrode Arrays for Scalable Intracellular Electrophysiology. Acc. Chem. Res. 2018, 51, 600-608.

(11) Angle, M. R.; Cui, B.; Melosh, N. A. Nanotechnology and neurophysiology. Curr. Opin. Neurobiol. 2015, 32, 132-140.

(12) Abbott, J.; et al. CMOS nanoelectrode array for all-electrical intracellular electrophysiological imaging. Nat. Nanotechnol. 2017, 12, 460-466.

(13) Xie, C.; Lin, Z.; Hanson, L.; Cui, Y.; Cui, B. Intracellular recording of action potentials by nanopillar electroporation. Nat. Nanotechnol. 2012, 7, 185-190.

(14) Lin, Z. C.; Xie, C.; Osakada, Y.; Cui, Y.; Cui, B. Iridium oxide nanotube electrodes for sensitive and prolonged intracellular measurement of action potentials. Nat. Commun. 2014, 5, 3206.

(15) Lin, Z. C.; et al. Accurate nanoelectrode recording of human pluripotent stem cell-derived cardiomyocytes for assaying drugs and modeling disease. Microsystems Nanoeng 2017, 3, 16080.

(16) Dipalo, M.; et al. Intracellular and Extracellular Recording of Spontaneous Action Potentials in Mammalian Neurons and Cardiac Cells with 3D Plasmonic Nanoelectrodes. Nano Lett. 2017, 17, 39323939.

(17) Fendyur, A.; Spira, M. E. Toward on-chip, in-cell recordings from cultured cardiomyocytes by arrays of gold mushroom-shaped microelectrodes. Front. Neuroeng. 2012, 5, 1-10.

(18) Spira, M. E.; Shmoel, N.; Huang, S. H. M.; Erez, H. Multisite attenuated intracellular recordings by extracellular multielectrode arrays, a perspective. Front. Neurosci. 2018, 12, 212. 
(19) Dipalo, M.; et al. Plasmonic meta-electrodes allow intracellular recordings at network level on high-density CMOS-multi-electrode arrays. Nat. Nanotechnol. 2018, 13, 965-971.

(20) Tian, B.; et al. Three-Dimensional, Flexible Nanoscale FieldEffect Transistors as Localized Bioprobes. Science (Washington, DC, U. S.) $2010,329,830-834$.

(21) Duan, X.; et al. Intracellular recordings of action potentials by an extracellular nanoscale field-effect transistor HHS Public Access Author manuscript. Nat. Nanotechnol. 2012, 7, 174-179.

(22) Zhao, Y., et al.Scalable ultrasmall three-dimensional nanowire transistor probes for intracellular recording. Nat. Nanotechnol. 2019, 14, 783.

(23) Dipalo, M.; et al. Cells Adhering to 3D Vertical Nanostructures: Cell Membrane Reshaping without Stable Internalization. Nano Lett. 2018, 18, 6100-6105.

(24) Almquist, B. D.; Melosh, N. A. Fusion of biomimetic stealth probes into lipid bilayer cores. Proc. Natl. Acad. Sci. U. S. A. 2010, 107, 5815-5820.

(25) Almquist, B. D.; Verma, P.; Cai, W.; Melosh, N. a. Nanoscale patterning controls inorganic-membrane interface structure. Nanoscale 2011, 3, 391-400.

(26) Almquist, B. D.; Melosh, N. A. Molecular structure influences the stability of membrane penetrating biointerfaces. Nano Lett. 2011, 11, 2066-2070.

(27) Verma, P.; Melosh, N. A. Gigaohm resistance membrane seals with stealth probe electrodes. Appl. Phys. Lett. 2010, 97, 033704.

(28) VanDersarl, J. J.; Renaud, P. Biomimetic surface patterning for long-term transmembrane access. Sci. Rep. 2016, 6, 32485.

(29) Capozza, R.; et al. Cell Membrane Disruption by Vertical Micro-/Nanopillars: Role of Membrane Bending and Traction Forces. ACS Appl. Mater. Interfaces 2018, 10, 29107-29114.

(30) Hanson, L.; Lin, Z. C.; Xie, C.; Cui, Y.; Cui, B. Characterization of the cell-nanopillar interface by transmission electron microscopy. Nano Lett. 2012, 12, 5815-5820.

(31) Desbiolles, B. X. E.; Bertsch, A.; Renaud, P. Ion beam etching redeposition for $3 \mathrm{D}$ multimaterial nanostructure manufacturing. Microsystems Nanoeng. 2019, 5, DOI: 10.1038/s41378-019-0052-7.

(32) Korhonen, T.; Hänninen, S. L.; Tavi, P. Model of excitationcontraction coupling of rat neonatal ventricular myocytes. Biophys. J. 2009, 96, 1189-1209.

(33) Rohr, S; Scholly, D M; Kleber, A G Patterned Growth of Neonatal Rat Heart Cells in Culture. Circ. Res. 1991, 68, 114-130.

(34) Dragas, J.; et al. In Vitro Multi-Functional Microelectrode Array Featuring 59760 Electrodes, 2048 Electrophysiology Channels, Stimulation, Impedance Measurement, and Neurotransmitter Detection Channels. IEEE J. Solid-State Circuits 2017, 52, 1576-1590.

(35) Ballini, M.; et al. A 1024-channel CMOS microelectrode array with 26,400 electrodes for recording and stimulation of electrogenic cells in vitro. IEEE J. Solid-State Circuits 2014, 49, 2705-2719.

(36) Heer, F.; et al. CMOS microelectrode array for the monitoring of electrogenic cells. Biosens. Bioelectron. 2004, 20, 358-366.

(37) Voskerician, G.; et al. Biocompatibility and biofouling of MEMS drug delivery devices. Biomaterials 2003, 24, 1959-1967.

(38) Miragoli, M.; Salvarani, N.; Rohr, S. Myofibroblasts induce ectopic activity in cardiac tissue. Circ. Res. 2007, 101, 755-758.

(39) Li, T.; Hu, W. Electrochemistry in nanoscopic volumes. Nanoscale 2011, 3, 166-176.

(40) Actis, P.; et al. Electrochemical nanoprobes for single-cell analysis. ACS Nano 2014, 8, 875-884.

(41) Baker, L. A. Perspective and Prospectus on Single-Entity Electrochemistry. J. Am. Chem. Soc. 2018, 140, 15549-15559.

(42) Kilic, T.; Navaee, F.; Stradolini, F.; Renaud, P.; Carrara, S. Organs-on-chip monitoring: sensors and other strategies. Microphysiological Syst 2018, 2, 1-2.

(43) Rohr, S.; Flückiger-Labrada, R.; Kucera, J. P. Photolithographically defined deposition of attachment factors as a versatile method for patterning the growth of different cell types in culture. Pfluegers Arch. 2003, 446, 125-132. 